\title{
Superior Mesenteric Vein Thrombosis Following Treatment of Refractory Immune Thrombocytopenic Purpura with Romiplostim
}

\author{
Dhaval Shah, John Nelson, Gurpreet Lamba, Sivamurthy Kyathari and Karen Seiter*
}

Division of Hematology/Oncology, New York Medical College, Valhalla, New York 10595, USA

\begin{abstract}
Romiplostim is a thrombopoietin receptor agonist approved for the treatment of thrombocytopenia in patients with chronic idiopathic thrombocytopenic purpura (ITP) who had an insufficient response to corticosteroids, immunoglobulins, or splenectomy. Although thrombotic and embolic complications have been reported in patients receiving romiplostim, these have generally involved coronary artery or cerebral vascular events. Currently we report a patient with severe ITP treated with romiplostim who developed mesenteric venous thrombosis after a hemicolectomy to resect colon cancer.
\end{abstract}

Keywords: ITP, Superior mesenteric vein thrombosis, romiplostim.

\section{INTRODUCTION}

Front line therapy of chronic immune thrombocytopenic purpura (ITP) includes corticosteroids and intravenous immunoglobulin [1, 2]. Although $60-70 \%$ of patients respond to such treatments, the disease recurs in $10-30 \%$. Treatment options for relapsed patients include splenectomy and/or rituximab [3, 4]. Romiplostim is a thrombopoietin receptor agonist that was recently approved by the United States Federal Drug Administration (FDA) for the treatment of thrombocytopenia in patients with chronic ITP who had an insufficient response to corticosteroids, immunoglobulins, or splenectomy [5]. The most common toxicities include arthralgia, dizziness, insomnia and myalgia. Although thrombotic and embolic complications have been reported in patients receiving romiplostim, these have generally involved coronary artery or cerebral vascular events [5-7]. These reports are summarized in Table 1. Currently we report a patient with severe secondary immune thrombocytopenia treated with romiplostim who developed mesenteric venous thrombosis after a hemicolectomy to resect a colon cancer.

A 77 year old woman presented to another hospital with melanotic stools. She had a history of hypertension, stroke, chronic hepatitis B and C infection, hypothyroidism, and hypercholesterolemia. Medications upon admission included aspirin and clopidogrel $75 \mathrm{mg}$ daily. The physical examination was significant for mild splenomegaly. The complete blood count (CBC) showed a white blood count of $5,000 / \mathrm{mm}^{3}$ with an unremarkable differential, a hemoglobin of $10.1 \mathrm{~g} / \mathrm{dl}$ and a platelet count of $1,000 / \mathrm{mm}^{3}$. Bone marrow aspiration and biopsy revealed a cellular marrow with trilineage maturation, erythyroid hyperplasia and mild megaloblastic changes. There was normal myeloid and megakaryocytic maturation. The blast count was $<1 \%$ and

*Address correspondence to this author at the Room 250 Munger Pavilion, New York Medical College, Valhalla, New York, 10595, USA; Tel: 914 493 7514; Fax: 914594 4420; E-mail: karen_seiter@nymc.edu the cytogenetics subsequently were determined to be normal. She received intravenous immunoglobulin $(500 \mathrm{mg} / \mathrm{kg}$ daily) for 4 days and prednisone $60 \mathrm{mg}$ daily. Despite aggressive treatment for suspected immune thrombocytopenia, on hospital day 11 her platelet count was still $3,000 / \mathrm{mm}^{3}$. She continued to have large melanotic stools despite numerous packed red cell and platelet transfusions. She then received romiplostim, $1 \mathrm{mcg} / \mathrm{kg}$, and was transferred to our facility for further management.

Upon admission the patient was critically ill with life threatening gastrointestinal bleeding. The white count was $9,700 / \mathrm{mm}^{3}$, the hemoglobin was $10.3 \mathrm{~g} / \mathrm{dl}$ and the platelet count was $3,000 / \mathrm{mm}^{3}$. The prothrombin time was 15.2 (normal PT 9.8-12.0 seconds) and the partial thromboplastin time (PTT) was prolonged at 48.0 seconds (normal PTT $\leq 31$ seconds). The PTT did not correct in a mixing study; a lupus anticoagulant titer was initially weakly positive at 1.2:1. The patient continued to have melanotic stools. She was treated with steroids and one dose of rituximab, $375 \mathrm{mg} / \mathrm{m}^{2}$. A bleeding scan demonstrated increased radiotracer uptake in the cecum. Abdominal ultrasound revealed a cirrhotic liver with patent hepatic and portal veins; the spleen was mildly enlarged at $13.8 \mathrm{~cm}$. Four days after admission the patient received a second course of intravenous immunoglobulin $(400 \mathrm{mg} / \mathrm{kg} / \mathrm{dose})$ for 5 days and a second weekly dose of romiplostim $(2 \mathrm{mcg} / \mathrm{kg})$. Colonoscopy revealed a friable ulcerated tumor in the cecum with active hemorrhage. The platelet count remained low at $19,000 / \mathrm{mm}^{3}$. The patient then received aminocaproic acid and continued on steroids. A week after admission she received a second dose of rituximab. Despite this treatment, the platelet count was only $21,000 / \mathrm{mm}^{3}$. Romiplostim was increased to $3 \mathrm{mcg} / \mathrm{kg}$ and after two more doses, the platelet count increased to $128,000 / \mathrm{mm}^{3}$. Prednisone was decreased to $20 \mathrm{mg}$ daily. Three weeks after admission to our facility, with a platelet count of $153,000 / \mathrm{mm}^{3}$, a laparoscopic assisted right hemicolectomy and partial omentectomy was performed. Pathology revealed a T2N0M0 moderately differentiated adenocarcinoma of the cecum. Postoperatively the platelet count increased to $189,000 / \mathrm{mm}^{3}$. The fifth dose of 
Table 1. Thrombosis in Patients Treated with Romiplostim

\begin{tabular}{|c|c|c|c|c|c|c|}
\hline Event & Refs. & Age/Sex & Risk Factors & Severity* & Rx Duration (Days) & Platelet Count $\left(/ \mathrm{mm}^{3}\right)$ \\
\hline Popliteal artery thrombosis & {$[5]$} & $82 / \mathrm{NA}$ & Yes $\dagger$ & NA & NA & 111,000 \\
\hline Myocardial infarction & {$[7]$} & $85 / \mathrm{F}$ & NA & 4 & 60 & NA \\
\hline Portal Vein thrombosis & {$[7]$} & $44 / F$ & NA & 3 & 823 & NA \\
\hline Transverse Sinus thrombosis & {$[7]$} & $63 / \mathrm{M}$ & NA & 3 & 363 & NA \\
\hline Thrombosis ${ }^{\S}$ & {$[7]$} & $57 / \mathrm{F}$ & NA & 4 & 95 & NA \\
\hline
\end{tabular}

Refs., reference; Rx, treatment; * Severity: 1, mild; 2, moderate; 3, severe; 4, life-threatening; 5, fatal.

$\dagger$ Extensive peripheral vascular disease, atrial fibrillation, history of radial artery thrombectomy. $\$$ Cerebrovascular disease, Congestive heart failure, diabetes and hypertension. $\S$ Thrombosed inflammatory fibrosis (preferred term thrombosis) was diagnosed at the site of a central line place13 years previously for blood draws because of poor vascular access.

romiplostim was decreased to $2 \mathrm{mcg} / \mathrm{kg}$ and prednisone was decreased to $10 \mathrm{mg}$ daily. One week postoperatively the platelet count abruptly dropped to $21,000 / \mathrm{mm}^{3}$. Steroids were increased and the weekly romiplostim dose was returned to $3 \mathrm{mcg} / \mathrm{kg}$ for her sixth dose. Two days later the platelet count increased to $56,000 / \mathrm{mm}^{3}$ and the steroids were decreased. Prior to the seventh dose of romiplostim, the platelet count had increased to $160,000 / \mathrm{mm}^{3}$. The patient complained of severe abdominal pain. CT scan of abdomen demonstrated an occlusive venous thrombosis in the superior mesenteric vein terminating at the confluence with the splenic vein. She was then treated with full dose heparin. Her abdominal pain improved and heparin was changed to warfarin. The platelet count remained above $150,000 / \mathrm{mm}^{3}$, reaching a maximum of $253,000 / \mathrm{mm}^{3}$ and she did not receive any further doses of romiplostim. Evaluations of protein $\mathrm{C}$, protein $\mathrm{S}$, prothrombin gene 20210 , and factor VIII were normal. A repeat lupus anticoagulant titer was strongly positive at 2.68:1 (normal titer $<1.2: 1$ ).

Within days of initial discharge, the patient was readmitted with abdominal pain and fever. A second laparotomy revealed abdominal fluid collections with polymicrobial infection. The patient died of septic shock with no further thrombotic issues.

\section{DISCUSSION}

Traditionally ITP was thought to result from production of antiplatelet antibodies which cause peripheral destruction of platelets. Recently, additional pathogenetic mechanisms have been discovered including decreased platelet production and an inappropriately low serum thrombopoietin level [8]. These data prompted the development of pharmacologic TPO-mimetic agents for these patients. Early studies of a polyethylene glycol-conjugated recombinant human megakaryocyte growth and development factor, a recombinant thrombopoietin, were associated with the development of antibodies which cross-reacted with endogenous thrombopoietin, causing persistent prolonged thrombocytopenia $[9,10]$.

Romiplostim is a second generation TPO-mimetic agent; it is a recombinant antibody with four c-mpl binding peptides linked to an immunoglobulin Fc carrier domain [11]. Romiplostim was approved for treatment of refractory ITP based on the results of two parallel phase III double blind, placebo controlled clinical trials in which patients (with or without prior splenectomy) received romiplostim weekly for 24 weeks. The primary end-point in both studies was a sustained increase in platelet count. Overall bleeding and thrombotic events were similar in both the romiplostim and placebo groups [5]. Thrombosis occurred in one patient assigned to placebo (pulmonary embolism) and in two assigned to romiplostim (popliteal artery thrombosis and a cerebrovascular event, respectively). During the extension study, 7 additional thrombotic events occurred in four patients including coronary artery occlusion, calf vein thrombosis and two patients with multiple events. One patient had portal vein thrombosis but no other details are available. Seven of the ten thromboembolic events occurred at platelet counts below the median peak platelet count of $167,000 / \mathrm{mm}^{3}$ for all patients treated with romiplostim in both the phase III and extension studies [6,7].

Our patient's platelet count was exceptionally labile reaching a peak of $154,000 / \mathrm{mm}^{3}$ prior to surgery and falling to $21,000 / \mathrm{mm}^{3}$ post operatively. The highest platelet count was $253,000 / \mathrm{mm}^{3}$ five days after she was diagnosed with superior mesenteric vein thrombosis. The platelet count was monitored daily and the FDA approved dosing was followed. It is recommended that romiplostim be discontinued if the platelet count is greater than $400,000 / \mathrm{mm}^{3}$, a platelet count which our patient never achieved. Our patient also had many coexisting risk factors for venous thrombosis. The patient was bed ridden and had recently undergone major abdominal surgery to resect an adenocarcinoma of the colon. She had received multiple courses of high dose steroids and a short course of aminocaproic acid in an attempt to treat her lifethreatening hemorrhage. There was underlying liver cirrhosis which is associated with increased abdominal venous thrombosis [12]. The patient also met criteria for antiphospholipid syndrome with a lupus anticoagulant. We suspect that the combination of these events led to her superior mesenteric vein thrombosis. It may be that special care should be used when romiplostim is given in the setting of multiple risk factors for venous thrombosis. It is possible that a lower platelet count should be targeted in these individuals, however it should also be noted that as soon as our patient's romiplostim dose was decreased her platelet count dropped precipitously. Of note, patients with active malignancies were excluded from the Phase III trial. As more complex patients are treated outside the setting of a 
clinical it is anticipated that additional toxicities will be uncovered.

\section{ACKNOWLEDGEMENT}

None declared.

\section{CONFLICT OF INTEREST}

None declared.

\section{REFERENCES}

[1] Provan D, Stasi R, Newland AC, et al. International consensus report on the investigation and management of primary immune thrombocytopenia. Blood 2010; 115(2): 168-86.

[2] Cines DB, McMillan R. Management of adult idiopathic thrombocytopenic purpura. Ann Rev of Med 2005; 56: 425-42.

[3] Kojouri K, Vesely SK, Terrell DR, George JN. Splenectomy for adult patients with idiopathic thrombocytopenic purpura: a systematic review to assess long-term platelet count responses, prediction of response, and surgical complications. Blood 2004; 104(9): 2623-34.

[4] Medeot M, Zaja F, Vianelli N, et al. Rituximab therapy in adult patients with relapsed or refractory immune thrombocytopenic purpura: longterm follow-up results. Eur J Haematol 2008; 81(3): $165-9$.

[5] Kuter DJ, Bussel JB, Lyons RM, et al. Efficacy of romiplostim in patients with chronic immune thrombocytopenic purpura: a double- blind randomized controlled trial. javascript:AL_get(this, 'jour', 'Lancet.'); Lancet 2008; 371(9610): 395-403.

[6] Bussel JB, Kuter DJ, Pullarkat V, Lyons RM, Guo M, Nichol JL. Safety and efficacy of long-term treatment with romiplostim in thrombocytopenic patients with chronic ITP. Blood 2009; 113(10): 2161-71.

[7] Gernsheimer TB, George JN, Aledort LM, et al. Evaluation of Bleeding and Thrombotic Events during Long-Term Use of Romiplostim in Patients with Chronic Immune Thrombocytopenic Purpura. J Thromb Haemost 2010; 8(6): 1372-82.

[8] Nugent D, McMillan R, Nichol JL, Slichter SJ. Pathogenesis of chronic immune thrombocytopenia: increased platelet destruction and/or decreased platelet production. Br J Haematol 2009; 6(6): 585-96.

[9] Nomura S, Dan K, Hotta T, Fujimura K, Ikeda Y. Effects of pegylated recombinant human megakaryocyte growth and development factor in patients with idiopathic thrombocytopenic purpura. Blood 2002; 100(2): 728-30.

[10] Li J, Yang C, Xia Y, et al. Thrombocytopenia caused by the development of antibodies to thrombopoietin. Blood 2001; 98(12): 3241-8.

[11] Bussel JB, Kuter DJ, George JN, et al. AMG 531, a Thrombopoiesis-Stimulating Protein, for Chronic ITP. N Engl J Med 2006; 355(16): 1672-81.

[12] Amitrano L, Guardascione MA, Brancaccio V, et al. Risk factors and clinical presentation of portal vein thrombosis in patients with liver cirrhosis. J Hepatol 2004; 40(5): 736-41.

Received: August 11, 2011 